\section{Index-guiding liquid-core photonic crystal fiber for solution measurement using normal and surface- enhanced Raman scattering}

\author{
Yun Han, ${ }^{a}$ Member SPIE, Maung Kyaw Khaing Oo, ${ }^{a}$ \\ Yinian Zhu, ${ }^{a}$ Limin Xiao, ${ }^{b}$ M. Suleyman Demohan, \\ Wei Jin, ${ }^{b}$ and Henry Du, ${ }^{a}$ MEMBER SPIE \\ ${ }^{a}$ Stevens Institute of Technology, Department of Chemical, \\ Biomedical and Materials Engineering, Hoboken, New \\ Jersey 07030 \\ E-mail: hdu@stevens.edu \\ ${ }^{\mathrm{b}}$ The Hong Kong Polytechnic University, Department of \\ Electrical Engineering, Kowloon, Hong Kong
}

Abstract. We have explored the use of index-guiding liquidcore (LC) photonic crystal fiber (PCF) as a robust platform for measurements of solutions of trace volume using normal and surface-enhanced Raman scattering (SERS). The LC PCF was fabricated by selectively sealing the cladding air channels at the distal ends of a hollow-core PCF while leaving the center core open, using a fusion splicer. Utilizing a 30-cm-long LC PCF with the entire center core filled with the $\sim 0.1-\mu \mathrm{L}$ solution of interest, we have obtained normal Raman spectra of water, ethanol, and $1 \mathrm{vol} \%$ ethanol in water. Sensitive and reproducible SERS detection of $1.7 \times 10^{-7} \mathrm{M}$ thiocyanate anions $(\approx 14 \mathrm{ppb}$ of $\mathrm{NaSCN})$ in water has also been achieved. () 2008 Society of Photo-Optical Instrumentation Engineers.

[DOI: 10.1117/1.2902463]

Subject terms: index-guiding liquid-core photonic crystal fiber; hollow-core photonic crystal fiber; Raman scattering; surfaceenhanced Raman scattering; sensing; detection.

Paper 070866 L received Oct. 23, 2007; accepted for publication Jan. 21, 2008; published online Apr. 14, 2008.

The combination of waveguide characteristics and unique microstructure feature in the form of axially aligned air channels along the entire fiber length makes photonic crystal fiber (PCF) a potentially robust and versatile chemical and biological sensing and detection platform. Indeed, PCFs have been increasingly explored utilizing sensing schemes based principally on absorption, ${ }^{1,2}$ fluorescence, ${ }^{3}$ and Raman scattering. ${ }^{4,5}$ Raman scattering is a particularly attractive sensing strategy due to its inherent capability for molecular fingerprinting. Surface-enhanced Raman scattering (SERS $)^{6-10}$ further enhances the prospect of integrated PCF and SERS platforms for ultratrace sensing and identification of analytes of interest in liquid or gas medium.

There exist two general types of PCFs: ${ }^{1}$ (1) solid-core (SC), with light guiding in the core via total internal reflectance due to index contrast between the SC and the air cladding, and (2) hollow-core (HC), with light guiding in the core via band-gap confinement of a carefully configured periodic air-cladding structure. Raman measurements using

0091-3286/2008/\$25.00 @ 2008 SPIE
SC PCF can be realized via interaction of the evanescent field with the analyte in the air channels immediately surrounding the light-guide SC. This scheme gives a rise to strong intrinsic Raman scattering in the core substance (silica), which may produce spectral interference. Alternatively, Raman detection using HC PCF can be achieved through direct light excitation of the analyte in the $\mathrm{HC}$ with silica Raman scattering contributing only very weakly to the background. Ability to selectively infiltrate solution phase into the center air hole of $\mathrm{HC} \mathrm{PCF}^{12-14}$ opens up the possibility of using a liquid-filled core as an index-contrast light guide for solution sensing and measurements. ${ }^{15,16}$

Yan et al. ${ }^{4}$ explored $\mathrm{Au}$ nanoparticle-coated $\mathrm{HC}$ PCF probing tips (1 to $3 \mathrm{~cm}$ in length) for SERS measurement of rhodamine $\mathrm{B}$ on drying of a $10^{-5} \mathrm{M}$ solution. The detection was carried out using backscattering geometry at the opposite end of a 5-cm-long fiber with the considerable Raman intensity of silica as spectral background. Zhang et al. ${ }^{5}$ reported the use of a $10-\mathrm{cm}$-long $\mathrm{HC}$ PCF with the $\sim 1$-cm tip of the center hole filled with analyte solutions mixed with Ag nanoparticles for SERS measurements of rhodamine 6G, human insulin, and tryptophan $\left(10^{-4}\right.$ to $\left.10^{-5} \mathrm{M}\right)$. The liquid-core (LC) approach explored by the authors was a novel one, but the short liquid filling length, particularly the utilization of the remaining 9-cm-long HC PCF for transmission of backscattered SERS, unnecessarily restricts the window of the transmitted Raman spectrum, due to the relatively narrow transmission band of the band-gap fiber.

We report in this letter the investigation of indexguiding LC PCF as a platform for measurements on water, ethanol, and their solutions using normal Raman scattering and for ultratrace detection of thiocyanate anions in $\mathrm{Ag}$ nanoparticle colloidal solution using SERS. There are four major differentiating features in our work, compared with the related published studies: (1) the solution phase as the index-guiding LC through the entire fiber length $(30 \mathrm{~cm}$ here) without band-gap confinement; (2) data acquisition via transmission geometry; (3) minimal background interference from silica Raman scattering, often a dominant feature in SC fiber geometry; and (4) demonstrated ultrasensitivity due to strong light-analyte overlap over a long interaction length.

The HC PCF purchased from Crystal-fiber A/S (Model HC19-1550-01) has a HC diameter of $\approx 20 \mu \mathrm{m}$ surrounded by a fine array of air channels of average diameter $\approx 3.5 \mu \mathrm{m}$. The band gap of this fiber is centered at a wavelength of $1550 \mathrm{~nm}$. Shown in Fig. 1(a) is a scanning elec-

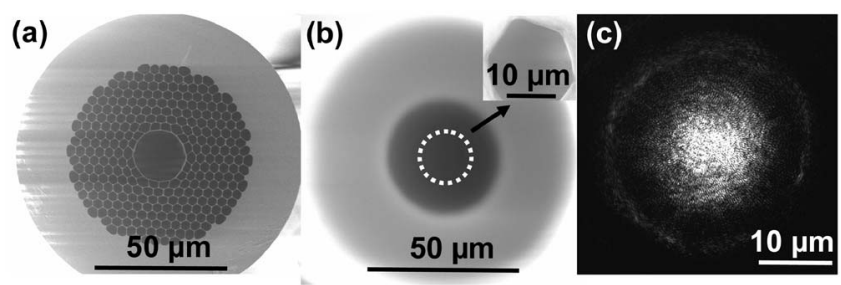

Fig. 1 Cross-sectional SEM micrograph of HC PCF (a) as received and (b) after splice-fusing closure of the cladding air channel at the distal end. Inset is an SEM micrograph of the open HC. (c) Nearfield image of the light intensity distribution in water-filled LC PCF at $632.8 \mathrm{~nm}$. 


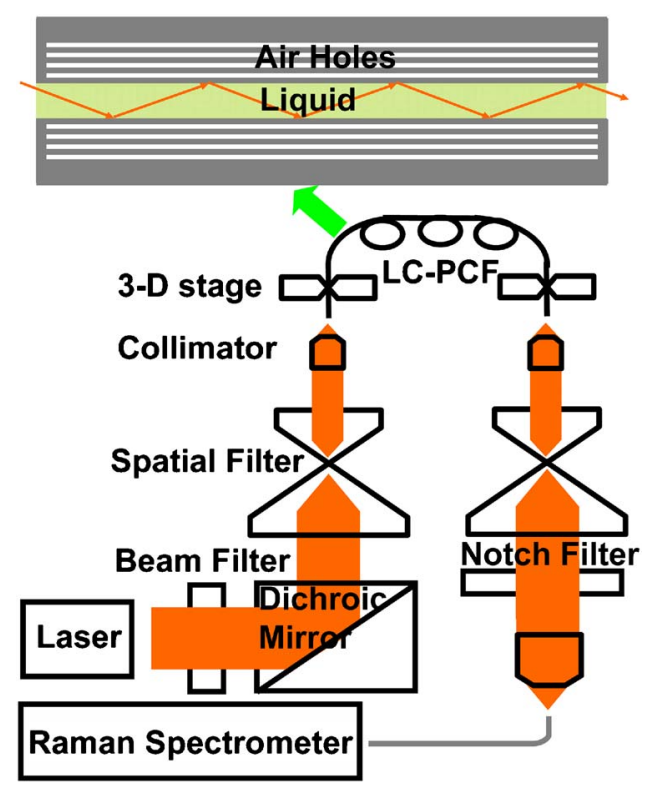

Fig. 2 Schematic illustrations of light guiding in the liquid core by total internal reflectance in a LC PCF (top) and a fiber Raman setup (bottom). (Color online only.)

tron microscopy (SEM) image of the cross section of the as-received HC PCF. The cladding air channels at the distal ends of a carefully cleaved 30-cm-long HC PCF were selectively sealed while leaving the center hole open using a fusion splicer (Ericsson FSU-995), following the strategy described elsewhere. ${ }^{13,14}$ Figure 1(b) illustrates the crosssection SEM of a HC PCF after splice fusion of the distal cladding air channels. The open $\mathrm{HC}$ is highlighted in the image due to insufficient contrast in the SEM image between the core region and the sealed air cladding area; the inset of Fig. 1(b) shows the dimension of the open core.

Filling of solution phase into the $\mathrm{HC}$ of the $\mathrm{HC} \mathrm{PCF}$ with cladding air channels sealed at the distal ends was conducted via liquid flow in the microfluidic hollow air core driven by a pressure differential. Specifically, one end of the fiber was immersed in the solution of interest inside a custom-made pressure cell, whereas the other end was left in the ambient air. A duration of 5 min was sufficient to allow complete solution filling in the $\mathrm{HC}$ at a chamber pressure of $200 \mathrm{psi}$ (nitrogen), resulting in a LC PCF with $\sim 0.1-\mu \mathrm{L}$ liquid phase as the light guide due to index contrast between the high-index liquid and the low-index air cladding. The light-guiding nature of the LC PCF is illustrated in Fig. 1(c), near-field image of the light intensity distribution from a water-filled LC PCF using transmission geometry at $632.8 \mathrm{~nm}$. The LC PCF platform, due to using the solution itself as the light-guiding medium, allows strong light-analyte interaction and Raman signal accumulation throughout the entire liquid-filled fiber length. Liquid filling effectively converts light guidance from bandgap confinement in the original HC PCF to total internal reflectance in the LC PCF, greatly expanding the spectral range that can be collected using the latter platform.

Shown in Fig. 2 is the setup used for the Raman measurements of LC PCF, with the schematic on the top depicting the total internal reflectance in LC PCF. Specifically, the LC PCF was mounted on the measurement station with

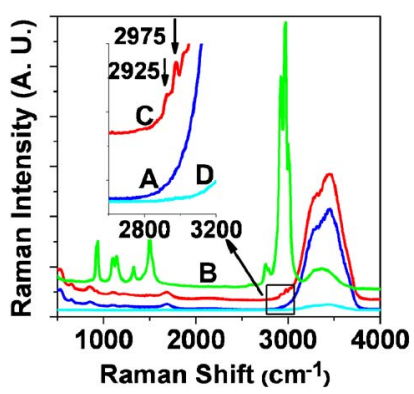

(a)

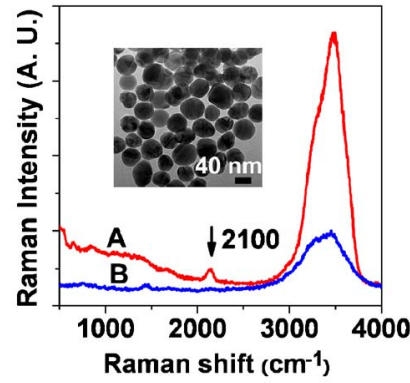

(b)
Fig. 3 (a) Normal Raman spectra acquired using a LC PCF filled with Milli-Q water $(A)$, pure ethanol $(B)$, and $1 \%(v / v)$ ethanol in Milli-Q water (C), and using a glass vial containing $1 \%(\mathrm{v} / \mathrm{v})$ ethanol in Milli-Q water (D). (b) SERS spectra obtained using a LC PCF filled with an analyte solution of $1.7 \times 10^{-7} \mathrm{M} \mathrm{SCN}^{-}$containing colloidal Ag nanoparticles (A) and using a glass vial filled with the same analyte solution (B). The Raman spectra were all taken using an excitation wavelength of $632.8 \mathrm{~nm}, 2.1 \mathrm{~mW}$, acquisition time $20 \mathrm{~s}$. Inset is a TEM micrograph of the Ag nanoparticles. (Color online only.)

precision 3-D microstages to align one end of the fiber to the excitation light source $(632.8 \mathrm{~nm})$ and to couple the other end to a liquid-nitrogen-cooled CCD Raman spectrometer. Normal Raman measurements were acquired using a $\sim 30-\mathrm{cm}$ LC PCF filled with Milli-Q water, ethanol, and their solution mixture. Illustrated in spectra $\mathrm{A}$ and $\mathrm{B}$ in Fig. 3(a) are respective Raman spectra of Milli-Q water and ethanol taken with a laser source at $632.8 \mathrm{~nm}, 2.1 \mathrm{~mW}$, and an acquisition time of $20 \mathrm{~s}$ using the LC PCF configuration. The spectra, from just $\sim 0.1 \mu \mathrm{L}$ of liquid, are clearly representative of water and ethanol, indicating the suitability of the LC PCF platform for Raman measurements of minute volumes of a liquid phase.

Note that Raman signal from silica in the spectral range of 500 to $1500 \mathrm{~cm}^{-1}$ constitutes only a weak background, suggesting strongly that the Raman scattering in our work is a result of direct interaction of the excitation light within the LC. The weak silica signal most likely originates from evanescent-field interaction with the thin silica web of the air cladding surrounding the liquid light-guide core as well as from the propagating surface modes in the silica part of the air cladding structure coupled with the core mode. ${ }^{15,17}$ The presence of surface modes is confirmed by the ring pattern in the near-field image shown in Fig. 1(c).

The robustness of the LC PCF for normal Raman spectroscopy is also demonstrated in our measurement of $1 \mathrm{vol} \%$ ethanol in Milli-Q water, as revealed by the distinct spectral features of ethanol at 2925 and $2975 \mathrm{~cm}^{-1}$ originating from $\mathrm{C}-\mathrm{H}$ vibrations shown in spectrum $\mathrm{C}$ in Fig. 3(a). For comparison, conventional Raman measurement was also attempted using the same ethanol-Milli-Q-water solution contained in a glass vial under a similar condition of laser excitation and acquisition time. As depicted in spectrum D in Fig. 3(a), not only is the overall Raman intensity dramatically reduced for the water band around $3400 \mathrm{~cm}^{-1}$, but no features corresponding to ethanol can be observed (see enlargement in inset). The strong light-field overlap with the solution phase over a long path length testifies to the powerful normal Raman capability using the LC PCF as a measurement platform. 
For ultra trace Raman detection using LC PCF with sodium thiocyanate (NaSCN) as a model analyte, SERSactive Ag colloidal nanoparticles were first produced by a UV-assisted citrate reduction process following the standard recipe of Lee and Meisel. ${ }^{18}$ Included in Fig. 3(b) as an inset is a transmission electron microscopy (TEM) micrograph of the resultant Ag nanoparticles. The nanoparticles have an average diameter of $\approx 40 \mathrm{~nm}$. UV-visible absorption analysis of the colloidal Ag nanoparticles revealed a sharp plasmon peak at $420 \mathrm{~nm}$. A solution mixture of $\mathrm{NaSCN}$ and colloidal $\mathrm{Ag}$ nanoparticles $(\approx 2.4$ $\times 10^{9}$ particle $/ \mathrm{mL}$ in water) with $1.7 \times 10^{-7} \mathrm{M} \mathrm{SCN}^{-}$was subsequently prepared for the filling in the $\mathrm{HC}$ of the $\mathrm{HC}$ PCF for SERS measurements using the same instrument parameters as for the normal Raman measurements described. Shown in spectrum A in Fig. 3(b) is a representative SERS spectrum of the analyte solution using LC PCF. Spectral measurements could be carried out reproducibly. The SERS Raman spectrum (B) taken from the same solution contained in a glass vial using conventional Raman measurements (as already described) is included in the figure for direct comparison. In addition to the water band at $3400 \mathrm{~cm}^{-1}$, the spectrum (A) from the LC PCF platform shows a pronounced Raman peak at $2100 \mathrm{~cm}^{-1}$ from the analyte solution, characteristic of $\mathrm{SCN}^{-}$stretching vibration. In contrast, the conventional Raman measurement approach was unable to detect $\mathrm{SCN}^{-}$anions at the same concentration. The SERS results are consistent with those from normal Raman scattering measurements and show that LC PCF offers excellent prospect for SERS-based liquid-phase Raman spectroscopy.

In conclusion, we have shown that index-guiding LC PCF can be used as a robust sensing and analytical platform for normal Raman scattering measurements of solution phases and SERS detection of ultratrace analyte in a solution. Specifically, using the LC PCF platform, normal Raman scattering is sensitive to 1 vol\% ethanol in Milli-Q water. Using the same platform, SERS is sensitive to 1.7 $\times 10^{-7} \mathrm{M} \mathrm{SCN}^{-}(\approx 14 \mathrm{ppb}$ of NaSCN$)$ in aqueous solution. LC PCF proved to offer a capability far beyond the conventional Raman approach. The excellent potential of LC PCF stems from the light-guiding nature of the analyte medium itself and the strong light-analyte field overlap over the entire path length of the fiber platform.

\section{Acknowledgments}

This work was supported by the U.S. National Science Foundation under grant No. ECS-0404002 (the U.S. team) and the Hong Kong Special Administration Region Government through a Competitive Earmarked Research Grant PolyU 5243/04E (the Hong Kong team).

\section{References}

1. G. Pickrell, W. Peng, and A. Wang, "Random-hole optical fiber evanescent-wave gas sensing," Opt. Lett. 29, 1476-1478 (2004).

2. J. B. Jensen, L. H. Pedersen, P. E. Hoiby, L. B. Nielsen, T. P. Hansen, J. R. Folkenberg, J. Riishede, D. Noordegraaf, K. Nielsen, A. Carlsen, and A. Bjarklev, "Photonic crystal fiber based evanescentwave sensor for detection of biomolecules in aqueous solutions," Opt. Lett. 29, 1974-1976 (2004).

3. S. Smolka, M. Barth, and O. Benson, "Selectively coated photonic crystal fiber for highly sensitive fluorescence detection," Appl. Phys. Lett. 90, 111101-111103 (2007).

4. H. Yan, C. Gu, C. Yang, J. Liu, G. Jin, J. Zhang, L. Hou, and Y. Yao, "Hollow core photonic crystal fiber surface-enhanced Raman probe," Appl. Phys. Lett. 89, 204101-204102 (2006).

5. Y. Zhang, C. Shi, C. Gu, L. Seballos, and J. Z. Zhang, "Liquid core photonic crystal fiber sensor based on surface enhanced Raman scattering," Appl. Phys. Lett. 90, 153504 (2007).

6. M. Fleischmann, P. J. Hendra, and A. J. McQuillan, "Raman spectra of pyridine adsorbed at a silver electrode," Chem. Phys. Lett. 26, 163-166 (1974).

7. D. L. Jeanmaire and R. P. V. Duyne, "Surface Raman spectroelectrochemistry. Part I. Heterocyclic, aromatic, \& aliphatic amines adsorbed on the anodized silver electrode," J. Electroanal. Chem. 84 1-20 (1977).

8. M. G. Albrecht and J. A. Creighton, "Anomalously intense Raman spectra of pyridine at a silver electrode," J. Am. Chem. Soc. 99, 5215-5217 (1977)

9. S. Nie and S. R. Emory, "Probing single molecules and single nanoparticles by surface-enhanced Raman scattering," Science 275, 1102 1106 (1997).

10. K. Kneipp, Y. Wang, H. Kneipp, L. T. Perelman, I. Itzkan, R. R. Dasari, and M. S. Feld, "Single molecule detection using surfaceenhanced Raman scattering (SERS)," Phys. Rev. Lett. 78, 1667-1670 (1997).

11. J. C. Knight, "Photonic crystal fibres," Nature (London) 424, 847$851(2003)$

12. Y. Huang, Y. Xu, and A. Yariv, "Fabrication of functional microstructured optical fibers through a selective-filling technique," Appl. Phys. Lett. 85, 5182-5184 (2004).

13. K. Nielsen, D. Noordegraaf, T. Sørensen, A. Bjarklev, and T. P. Hansen, "Selective filling of photonic crystal fibres," J. Opt. A, Pure Appl. Opt. 7, L13-L20 (2005).

14. L. Xiao, W. Jin, M. S. Demokan, H. L. Ho, Y. L. Hoo, and Chunliu Zhao, "Fabrication of selective injection microstructured optical fibers with a conventional fusion splicer," Opt. Express 13, 9014-9022 (2005)

15. J. M. Fini, "Microstructure fibres for optical sensing in gases and liquids," Meas. Sci. Technol. 15, 1120-1128 (2004).

16. S. O. Konorov, C. J. Addison, H. G. Schulze, R. F. B. Turner, and M W. Blades, "Hollow-core photonic crystal fiber-optic probes for Raman spectroscopy," Opt. Lett. 31, 1911-1913 (2006).

17. C. M. Smith, N. Venkataraman, M. T. Gallagher, D. Müller, J. A West, N. F. Borrelli, D. C. Allan, and K. W. Koch, "Low-loss hollowcore silica/air photonic bandgap fibre," Nature (London) 424, 657659 (2003).

18. P. C. Lee and D. Meisel, "Adsorption and surface-enhanced Raman of dyes on silver and gold sols," J. Phys. Chem. 86, 3391-3395 (1982). 